\title{
Quantum Hall Transition in the Classical Limit
}

\author{
V. Gurarie ${ }^{a, b}$ and A. Zee ${ }^{a}$ \\ ${ }^{a}$ Institute for Theoretical Physics, University of California, Santa Barbara CA 93106-4030 \\ ${ }^{b}$ Department of Theoretical Physics, Oxford University, 1 Keble Road, Oxford OX1 3NP, United Kingdom
}

(October 29, 2018)

\begin{abstract}
We study the quantum Hall transition using the density-density correlation function. We show that in the limit $\hbar \rightarrow 0$ the electron density moves along the percolating trajectories, undergoing normal diffusion. The localization exponent coincides with its percolation value $\nu=\frac{4}{3}$. The framework provides a natural way to study the renormalization group flow from percolation to quantum Hall transition. We also confirm numerically that the critical conductivity of a classical limit of quantum Hall transition is $\sigma_{x x}=\sqrt{3} / 4$.
\end{abstract}

\section{INTRODUCTION}

Integer quantum Hall transition is a localization-delocalization transition experienced by a quantum mechanical particle moving in a two dimensional plane in a magnetic field perpendicular to the plane and in a random scalar potential $V(x)$. As the energy of the particle approaches its critical value, the particle becomes more and more delocalized. In spite of the fact that the problem has been studied numerically and experimentally for 20 years, it has resisted exact theoretical descriptions.

In this paper we examine a new method to probe the properties of the quantum Hall transitions recently introduced by Girvin et al [1]. We show that this method provides a natural framework in which the classical limit of quantum Hall transition (classical percolation) can be studied. We show, both numerically and analytically, that even in this classical limit the percolating particle exhibits normal diffusion, just as the quantum critical particle would. The critical exponent $\nu$ turns out to be equal to the percolation exponent $\frac{4}{3}$. In this framework, the renormalization group flow from percolation to the quantum Hall transition can be studied.

The method introduced in [1] is based on the introduction of the density-density correlation function

$$
G(x, t)=\sum_{n}\langle n|\rho(x, t) \rho(0)| n\rangle
$$

where $\rho$ is the density operator and the sum goes over the Hilbert space of states. This correlation function has a simple meaning. It is the probability that a quantum particle, localized at the origin at the initial moment in time, is found at the position $x$ at a time $t$. Indeed, set up a wave function of a particle localized at the origin,

$$
\psi(x)=\sum_{n} \psi_{n}^{*}(0) \psi_{n}(x),
$$

where $\psi_{n}$ are the eigenstates of the Hamiltonian of the problem. At time $t$ the wave function will be (we set $\hbar=1$ )

$$
\psi(x, t)=\sum_{n} \psi_{n}^{*}(0) \psi_{n}(x) e^{i E_{n} t},
$$

where $E_{n}$ are the eigenenergies. The probability is equal to

$$
|\psi(x, t)|^{2}=\sum_{m, n} \psi_{n}^{*}(0) \psi_{n}(x) \psi_{m}^{*}(x) \psi_{m}(0) e^{i\left(E_{n}-E_{m}\right) t} .
$$

The matrix elements of the density operator are

$$
\rho_{m, n}(x)=\psi_{m}^{*}(x) \psi_{n}(x) .
$$

It is therefore obvious that the correlation function

$$
G(x, t)=\sum_{m, n} \rho_{m, n}(x, t) \rho_{n, m}(0)
$$


is equal to the probability (4).

It sounds reasonable that such a correlation function, which tests how far a particle can go in a given amount of time, is useful when studying localization. In the localization literature, however, a different function is usually studied. It is called the spectral function and is given by the square of the single particle Green's function [2]

$$
S(x, \omega ; E)=\sum_{m, n} \frac{\psi_{m}^{*}(x) \psi_{m}(0) \psi_{n}(x) \psi_{n}^{*}(0)}{\left(E-\frac{\omega}{2}-i \epsilon-E_{n}\right)\left(E+\frac{\omega}{2}+i \delta-E_{m}\right)},
$$

with $\epsilon$ and $\delta$ infinitesimally small numbers. As is well known, the conductance of the system is simply related to the spectral function $S(x, \omega ; E)$. This function contains more information than the correlation $G$. It tells us how far a particle can go if it propagates along the state with a given energy $E$. The correlation function $G$ is related to the integral of $S$ over $E$,

$$
2 \pi G(x, \omega+i \epsilon+i \delta)=\int_{-\infty}^{\infty} d E S(x, \omega ; E)
$$

where $G(x, \omega)$ is a Fourier transform of $G(x, t)$. The last formula can be obtained by directly integrating (4) and (77).

One could argue that the density-density correlator $G(x, t)$ we just introduced would not give much information about the localization properties. Indeed, in a transition such as Anderson transition in $3 \mathrm{D}$, this function will always be long ranged due to the contributions of the delocalized states to (8). In contrast, the spectral function $S(x, \omega ; E)$ tests only the states at the energy $E$.

The insight of Girvin at al was that in quantum Hall transition only the centers of the Landau bands are believed to be delocalized. Moreover, the physics of quantum Hall transitions allows us to restrict the particle to the lowest Landau level. In practical terms, that means restricting the sum in (6) to go only over the states in the lowest Landau level. Then there is only one delocalized state. In (8) only the states whose localization length is larger than $x$ contribute to the integral over all the energies. Consequently the density-density correlation function includes in itself some useful information about quantum Hall transitions.

It follows from the scaling theory of phase transitions that the localization length $\xi(E)$ is divergent as we approach the localization transition with the exponent $\nu$,

$$
\xi(E) \propto\left|E-E_{c}\right|^{-\nu}
$$

where $E_{c}$ is the critical energy. It has also been argued [2] that a quantum particle in 2D at the localizationdelocalization transition undergoes normal diffusion, which in particular results in a finite critical conductivity. Heuristically people sometimes estimate the Fourier-transformed density-density correlator by an interpolating method based on (8). Until the particle hits the localization length, we assume that

$$
S(k, t ; E) \propto \exp \left(-D t \frac{k^{2}}{4}\right) \text {, if } D t \ll \xi^{2}(E),
$$

which describes diffusion with the diffusion constant $D$. This assumption is true only if $k \rightarrow 0$ [2]. The diffusion at small $k$ reflects the existence of critical conductivity in quantum Hall effect. On the other hand, at larger $k$ the motion of the particle becomes more complicated. That reflects the fact that critical conductance is not Ohmic and in fact dependent on the shape of the sample. Obviously, (10) will not work if the particle is deeply in the localized regime, when $\left|E-E_{c}\right| \gg E_{\max }$, with $E_{\max }$ describing the scale where (10) and (9) break down.

If we wait long enough,

$$
S(k, t ; E) \propto \exp \left(-\frac{k^{2}}{4} \xi^{2}(E)\right), \text { if } D t \gg \xi^{2}(E) .
$$

Roughly speaking, $G(x, t)$ can be estimated as

$$
G(k, t) \propto \int_{E_{c}-E_{\max }}^{E_{c}+E_{\max }} d E \exp \left(-\frac{k^{2}}{4\left(\frac{1}{D t}+\frac{1}{\xi^{2}(E)}\right)}\right),
$$

where the function being integrated interpolates between the limiting behavior (10) and (11). Using (90) and changing the variables $E=E_{c}+z t^{-\frac{1}{2 \nu}}$, we can estimate this integral to go as at 


$$
\begin{aligned}
& G(k, t)=t^{-\frac{1}{2 \nu}} \int_{0}^{t^{2 \nu} E_{\max }} d z \exp \left(-\frac{k^{2} t}{4\left(D^{-1}+z^{2 \nu}\right)}\right)= \\
& E_{\max }-t^{-\frac{1}{2 \nu}} \int_{0}^{t^{2 \nu} E_{\max }} d z\left[1-\exp \left(-\frac{k^{2} t}{4\left(D^{-1}+z^{2 \nu}\right)}\right)\right] \propto 1-t^{-\frac{1}{2 \nu}} g\left(k^{2} t\right),
\end{aligned}
$$

where $g$ is some complicated function. We used that the integral on the second line can be extended to infinity. $G(x, t)$ is diffusive at small $k$ (a function of $\frac{x^{2}}{t}$ ) with a prefactor which is $t$ dependent. Knowing the prefactor allows us to determine the critical exponent $\nu$. This formula has been tested numerically in [1] and was found to give values of $\nu$ for the quantum Hall transition around the value accepted by the community.

Imprecise as the derivation of this formula may seem, we will show later in this paper how it can be given a precise meaning in the classical limit.

\section{EQUATION OF MOTION}

Now we would like to demonstrate that, as was first shown in [1], the density-density correlation function $G(x, t)$, when projected to the lowest Landau level and in the presence of a scalar potential $V(x)$, satisfies a simple equation.

Choose the basis of states in the lowest Landau level as in (see [3] and references therein)

$$
\psi_{n}\left(z, z^{*}\right)=\frac{z^{n}}{\sqrt{\left(2 l^{2}\right)^{n} n ! \pi}} \exp \left(-\frac{z z^{*}}{4 l^{2}}\right),
$$

where, as always, $z=x+i y$, complex coordinates on the $2 \mathrm{D}$ plane and $l$ is the so called magnetic length. Consider the Fourier transformed density operator

$$
\rho_{k}=\exp \left[\frac{i}{2}\left(k z^{*}+k^{*} z\right)\right]
$$

where similarly to the complex coordinates $k=k_{x}+i k_{y}$. Let us project this operator to the lowest Landau level, following the methods developed in [4]. The operator consists of the product of two factors. One of them, exp $\left[\frac{i}{2} k^{*} z\right]$ is analytic in $z$ and so acts entirely within the lowest Landau level. As such, it does not have to be projected. Indeed, if we multiply one of the wave functions (14) of the lowest Landau level by this factor, the result can be reexpressed as a linear combination of other functions of (14). The other factor involves $z^{*}$ and therefore does not act within the lowest Landau level. It has to be projected. We note, however, that the operator

$$
\hat{z}=2 l^{2} \frac{\partial}{\partial z}+\frac{1}{2} z^{*}
$$

acts within the lowest Landau level,

$$
\hat{z} \psi_{n}\left(z, z^{*}\right)=\sqrt{2 l^{2} n} \psi_{n-1}
$$

Furthermore,

$$
\int d^{2} z\left(z^{*}\right)^{n}\left(z^{*}\right)^{s} z^{m} \exp \left(-\frac{z z^{*}}{2 l^{2}}\right)=\int d^{2} z\left(z^{*}\right)^{n} \exp \left(-\frac{z z^{*}}{4 l^{2}}\right)\left(2 l^{2} \frac{\partial}{\partial z}+\frac{1}{2} z^{*}\right)^{s}\left[z^{m} \exp \left(-\frac{z z^{*}}{4 l^{2}}\right)\right] .
$$

In other words,

$$
\left\langle n\left|\left(z^{*}\right)^{s}\right| m\right\rangle=\left\langle n\left|\left(2 l^{2} \frac{\partial}{\partial z}+\frac{1}{2} z\right)^{s}\right| m\right\rangle
$$

This allows us to write down the projected density operator as in

$$
\rho_{k}=\exp \left[\frac{i}{2} k \hat{z}\right] \exp \left[\frac{i}{2} k^{*} z\right] .
$$

Normally the density operators commute with each other, but the projected density operators do not. Multiplying two density operators together and using (16) we obtain 


$$
\rho_{k} \rho_{q}=\exp \left[\frac{l^{2}}{2}(k \cdot q+i k \times q)\right] \rho_{k+q},
$$

where $k \cdot q$ is the dot and $k \times q$ is the cross products of two vectors $\left(k_{x}, k_{y}\right)$ and $\left(q_{x}, q_{y}\right)$.

Finally we note that the Hamiltonian of a particle moving in a lowest Landau level in the presence of the scalar potential $V(x)$ is just that potential itself projected to the lowest Landau level,

$$
H=\int d x V(x) \rho(x)=\int d k V(k) \rho_{-k} .
$$

Therefore, the equation of motion for the operator $\rho$ is

$$
i \hbar \frac{\partial}{\partial t} \rho_{k}=\left[H, \rho_{k}\right]=\int d^{2} q 2 i \sin \left(\frac{l^{2}}{2} k \times q\right) V(k-q) \exp \left[-\frac{l^{2}}{2}\left(k^{2}-k \cdot q\right)\right] \rho_{q},
$$

where we restored the $\hbar$ factor. Clearly $G(k, t)=\operatorname{tr}\left(\rho_{k}(t) \rho_{-k}(0)\right)$ satisfies

$$
i \hbar \frac{\partial}{\partial t} G(k, t)=\int d^{2} q 2 i \sin \left(\frac{l^{2}}{2} k \times q\right) V(k-q) \exp \left[-\frac{l^{2}}{2}\left(k^{2}-k \cdot q\right)\right] G(q, t),
$$

with the boundary condition

$$
G(k, 0)=\operatorname{tr}(\rho(k) \rho(-k))=N \exp \left(-\frac{l^{2}}{2} k^{2}\right),
$$

$\mathrm{N}$ being the number of states in the lowest Landau level. Solving this equation allows us in principle to determine $G(k, t)$. Therefore, it encodes all the properties of quantum Hall transitions. Unfortunately, while this equation is simple to write down, solving it is not a trivial task.

\section{CLASSICAL LIMIT}

Let us consider the classical limit of the quantum Hall transition. The classical limit $\hbar \rightarrow 0$ is equivalent here to the limit $l \rightarrow 0$ since $l^{2} \propto \hbar$. In this limit, the equation (24) becomes

$$
\frac{\partial G(x, t)}{\partial t}=\frac{l^{2}}{\hbar} \epsilon_{i j} \partial_{i} V \partial_{j} G(x, t) .
$$

Since $l^{2} \propto \hbar$, the planck constant completely drops out. In what follows, we choose the units where $\hbar / l^{2}=1$.

The formal solution to this equation is straightforward to obtain:

$$
G(x, t)=\delta(x(t)-x),
$$

where $x(t)$ is a trajectory satisfying the following equation

$$
\frac{d x_{i}(t)}{d t}=\epsilon_{i j} \partial_{j} V(x(t))
$$

Studying the solutions to the equation (26) reduces to studying the trajectories satisfying (28).

The tangent to the trajectory $x(t)$ is always perpendicular to the gradient of the potential $V(x(t))$. Therefore, $V(x(t))$ is conserved along the trajectory. In other words, the particle moves along the equipotential lines, as shown on Fig. 1. The value of the potential along the trajectory becomes an important parameter $V_{0}=V(x(t))$ which can be assigned to each trajectory satisfying (28). In a way, the potential $V(x)$ can be thought of as a mountainous landscape with peaks and valleys. As water fills the valleys of the landscape to the height $V_{0}$, the equipotential lines of Fig 1 are the shapes of the lakes formed in this way. 


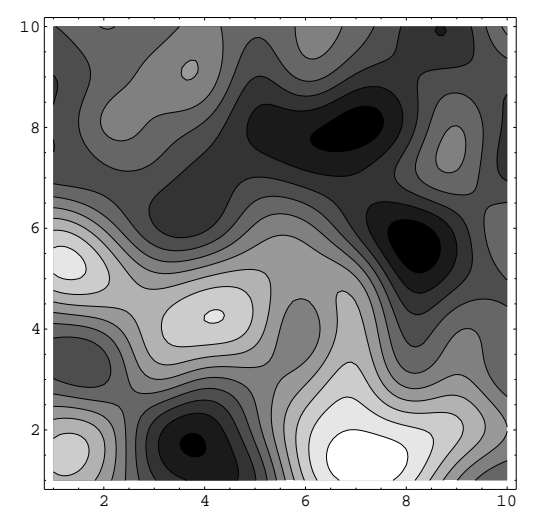

FIG. 1. Equipotential lines of a typical random potential $V(x)$. These are trajectory lines of the solutions to (28). The potential in this example is generated as a sum of Gaussian shapes located at the nodes of a square lattice with random amplitudes.

In this form the problem we study becomes equivalent to classical percolation [5]. Indeed, the potential $V(x)$ is random and independent in different points in space. We can consider the value of the potential at a point $x$ as a random variable. Take a particular trajectory $x(t)$ with a particular value of $V(x(t))=V_{0}$. Let us say that the point $x$ is "on" if $V(x)>V_{0}$ and "off" otherwise. Then the trajectory surrounds the clusters of the "on" sites. The trajectory becomes the so-called "hull" of the percolation cluster, and the area it surrounds is the percolation cluster itself (or the islands in the landscape analogy, for $\left.V_{0}>0\right)$. In the percolation problem, the site is "on" with a probability $p$. In our problem it can be expressed as in

$$
p=\int_{V_{0}}^{\infty} P(V) d V
$$

where $P(V)$ is the probability for the values of the potential at different points in space (sites). In this way, we recover the classical percolation picture of the quantum Hall transitions [6]. The electron density percolates along the equipotential lines of the scalar potential. The trajectories are closed. However, as $V_{0} \propto\left(p-\frac{1}{2}\right)$ approaches zero, the trajectories become more and more extended in space. Denoting by $\xi$ the root mean square distance between points on the trajectory, we say that the percolation hulls exhibit the delocalization transition, with $\xi \propto V_{0}^{-\nu}$.

Define the area of a cluster as

$$
A=\int d^{2} x \theta\left(V(x)-V_{0}\right),
$$

$\theta(x)=1$ when $x>0$ and $\theta(x)=0$ when $x<0$.

It turns out that the time it takes to go along the boundary of a cluster is just $d A / d V_{0}$, or

$$
T=\int d^{2} x \delta\left(V_{0}-V(x)\right) .
$$

This can be derived directly from (28). Alternatively, one can argue that (28) are the Hamiltonian equations, with $V$ being the Hamiltonian. Then $S$ is the "adiabatic invariant" and its derivative is obviously time.

From here one can conclude that, since the area of a cluster goes as $A \propto V_{0}^{-\gamma}$, the time goes as $T \propto V_{0}^{-\gamma-1}$, where $\gamma=\frac{43}{18}$ is another well known percolation exponent [5]. However, as we will see below, $T$ is not equal to the time it takes for a particle to go around the cluster. Rather it is much bigger having to do with the "swiss cheese" shape of a typical cluster. As a result, $T$ sums the time it takes go around the cluster, and around each hole in the cluster.

The classical percolation picture of the quantum Hall transitions was suggested a long time ago. It was subsequently demonstrated that it does not capture the physics of quantum Hall transitions. Indeed, the exponent $\nu$ computed in this way coincides with the its value for percolation $\nu=\frac{4}{3}[\nabla$, while the true value for $\nu$ is believed to be around $\nu=2.35 \approx \frac{7}{3}$. We can say that the classical density-density correlation function $G(x)$ forgets about the phase a quantum particle carries with itself. It was also known that the classical percolation picture can be derived as a classical limit of the Chalker-Coddington model [8], which is believed to lie in the same universality class as quantum Hall transitions.

Some time ago, F. Evers undertook a thorough numerical study of the solutions to the equations (28) at zero energy $V_{0}$, motived by classical limit of quantum Hall transition, in the paper [9]. Its results will be useful for us below. 
Now we are going to study percolation as a classical limit of quantum Hall transition. The approach we are using allows us to give a precise definition of what percolation is for quantum Hall transition, which quantum Hall quantities percolate in the classical picture and finally how they change as we allow for finite $l$ to account for the quantum effect.

\section{PERCOLATION}

To study the percolation limit, we suggest a lattice model of the equation (28). As a possible lattice model, we could have taken the classical limit Chalker-Coddington model [8]. This is the version of the model where a particle turns left or right at each node with a certain probability, instead of a certain quantum probability amplitude as in the original work [8]. However, to make a closer connection with percolation, we would like to consider a somewhat different model.

Consider a triangular lattice. Associate with each of its sites a random number $V$ drawn from a probability distribution $P(V)$. Now associate the points of the dual lattice with the path $x(t)$. Each path is assigned a number $V_{0}$, the value of the energy on this particular path. At each step the path moves in such a way that the potential of the left node of the bond the particle crosses is greater than $V_{0}$ which in turn is greater than the potential of the right node. For example, the particle on Fig 2 enters the triangle through the horizontal bond, as shown by the solid line. Therefore, $a>V_{0}>b$. If $c>V_{0}$, it would leave the triangle through the right bond, as shown by the dashed line. If $c<V_{0}$, then it would leave the triangle through the left bond.

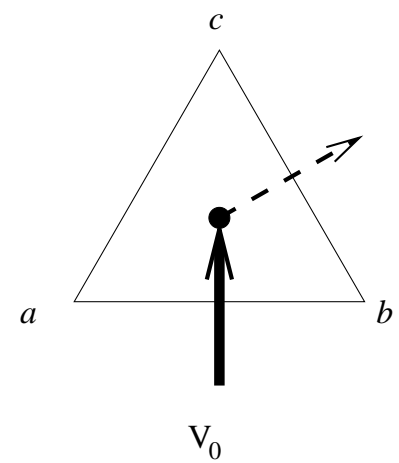

FIG. 2. The particle moves in such a way that the potential $c$ of the left node of the bond the particle crosses is greater than $V_{0}$ which in turn is greater than the potential $b$ of the right node.

The trajectory then becomes the hull of percolation clusters. A typical hull is shown on Fig 3 .

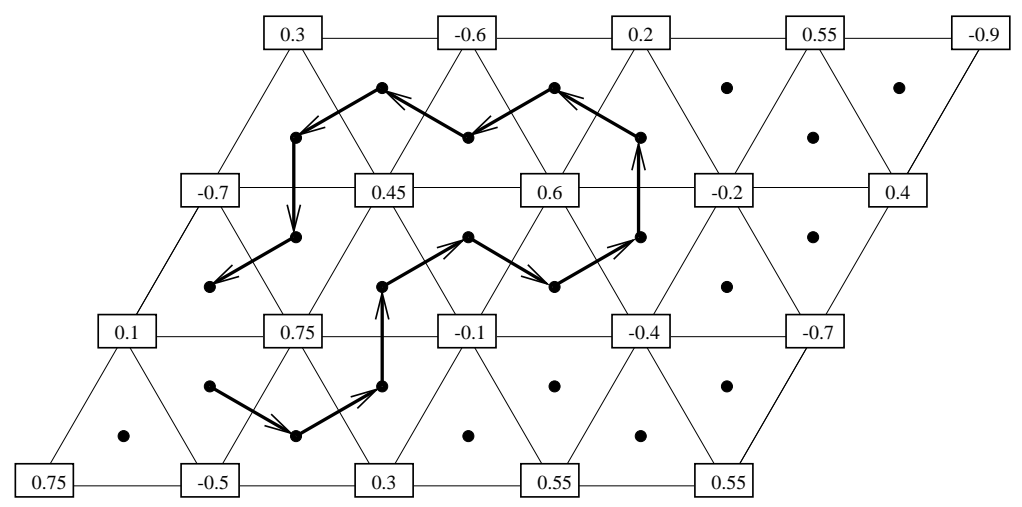

FIG. 3. A typical trajectory. The value of its potential $V_{0}=0.4$

The equation (26) also contains dynamical information. The velocity of the particle which follows (28) is proportional to the gradient of the potential. We model it by saying that the time it takes for a particle to traverse a bond of the triangular lattice is inversely proportional to the potential difference at the ends of the bond. For example, the time it takes to traverse the bond of the Fig. 2 (the process denoted by the dashed line) is $1 /(c-b)$.

Using this model, we performed extensive numerical simulations. In the simulations we assumed that the values of the potential at the nodes of the triangular lattice are random numbers uniformly distributed on the interval $[-1,1]$. 
The lattice size was 8500 by 8500 , and we discarded the trajectories which went outside the lattice. The typical trajectories were, however, of much smaller size, about 500. We discovered that the time it takes for a particle to go along a trajectory is essentially proportional to the trajectory's length. This is because the velocity of the trajectory remains roughly the same as $V_{0}$ is varied, having no singularity or zero as $V_{0}$ passes through 0 . Indeed, it is easy to estimate the average velocity by assuming that all the potentials at the corners of a triangle are random independent numbers. Then all we need to do is to average $1 /\left(V_{1}-V_{2}\right)$ where $V_{1}$ and $V_{2}$ are the values of the potential at the ends of the bond the trajectory is traversing. For the potential uniformly distributed on the interval $[-1,1]$ we obtain that the average velocity is very weakly $V_{0}$ dependent, with its value being $\log (4)$ when $V_{0}=0$. This was confirmed numerically. In fact, that coincides with the earlier observation in [9].

Interestingly, that allows us to find the time $T$ it takes for a particle to go around the cluster. The length of a trajectory is known to go as

$$
L \propto V_{0}^{-\gamma^{\prime}}
$$

where $\gamma^{\prime}=2$ [10]. Since the average velocity is only weakly $V_{0}$ dependent, we obtain that $T \propto V_{0}^{-2}$. That should be contrasted with the time it takes to go around the cluster and around each hole in the cluster which we found in the previous section.

Replacing time with length we studied $\overline{x^{2}(t)}$ where the average means averaging over different trajectories with the same value of $V_{0}$. In our simulations we averaged over about $10^{6}$ trajectories. Fig. A summarizes the results of the numerics.

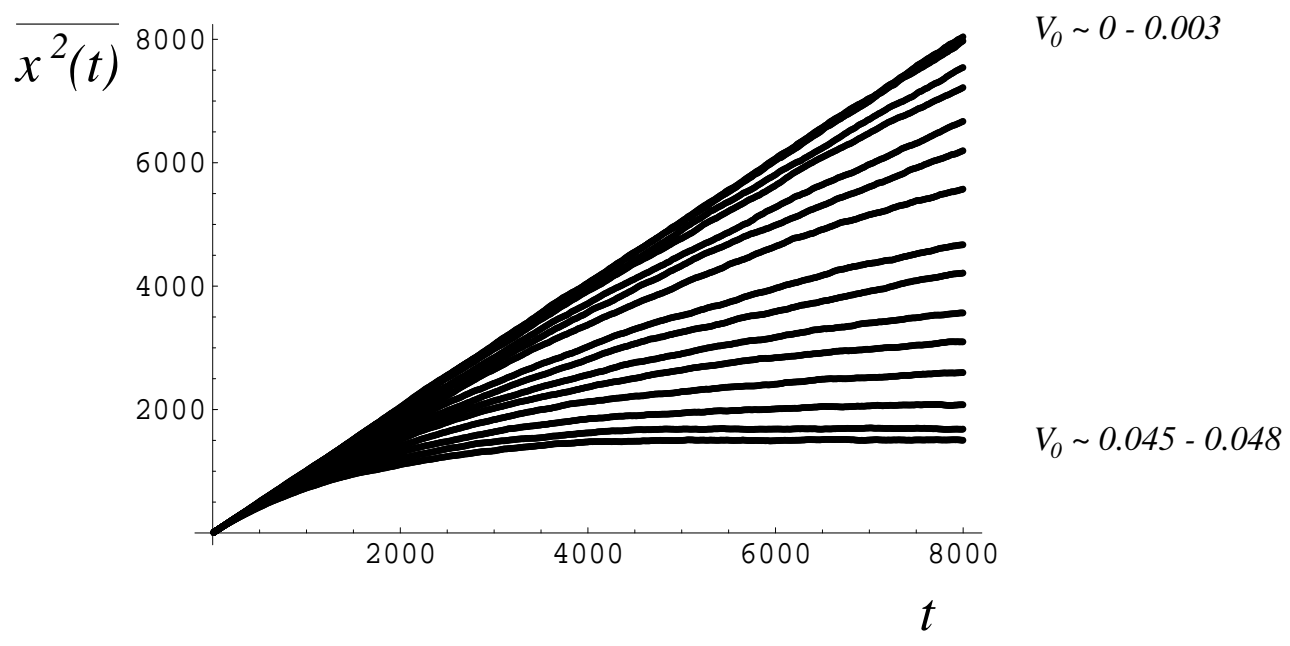

FIG. 4. $\overline{x^{2}(t)}$ computed for trajectories with various $V_{0}$. The highest line represents the lowest $V_{0}$. The potential at the nodes of the lattice is uniformly distributed from -1 to 1 . Time $t$ is simply the number of steps. $x^{2}$ is measured in units where the triangular lattice size of Fig. 3 is one.

It is clear that $\overline{x^{2}(t)}$ is a straight line

$$
\overline{x^{2}(t)}=D t, t \ll t^{*}
$$

until a certain time $t^{*}$ which varies with $V_{0}$. After that,

$$
\overline{x^{2}(t)}=\xi^{2}, t \gg t^{*}
$$

with the parameter $\xi$ depending on $V_{0}$ but not on time.

The length $\xi$ is none other than the localization (correlation) length of the percolation problem we introduced above. Indeed, if we wait long enough, the particle satisfying (28) starts winding around the closed trajectories such as on Fig. 1. Then $\overline{x^{2}(t)}$ measures the mean square distance between two points on the percolation cluster's hull which is the trajectory we study. So we can write down

$$
\xi \propto V_{0}^{-\frac{4}{3}}
$$

which we also confirmed by our numerical simulations. 
The numerical result that $\overline{x^{2}(t)} \propto t$ for $t<t^{*}$ shows that the particle undergoes a normal diffusion. While this is what is expected of a quantum particle in a quantum Hall transition [2], it is far less obvious that a classical particle should do the same. It is also not obvious how to explain this from a purely percolation point of view.

This result was previously obtained in [9] where $\overline{x^{2}(t)}$ for the trajectories at $V_{0}=0$ was studied. There it was shown that the diffusion was due to a "mysterious" cancellation of exponents in percolation. Perhaps, a certain scaling relation between those exists which leads to the cancellation, but at the moment we are not aware of it.

Now we would like to note that a convenient way to compute $\nu$ would be just summing all the trajectories of Fig. 16 together. As above, we expect this to be

$$
\sum_{V_{0}} \overline{x^{2}(t)}=\int d V_{0} P\left(V_{0}\right) \frac{1}{\frac{1}{D t}+V_{0}^{2 \nu}} \propto t^{1-\frac{1}{2 \nu}}
$$

Numerically this exponent is around 0.62 which corresponds to $\nu=1.30$, very close to the exact value. This method proves to be very convenient when studying quantum Hall transition in the quantum regime as well 11 .

Knowing $x(t)$ it is not difficult to compute $G(x, t)$ according to (27).

$$
G(k, t)=\sum_{V_{0}} \overline{\exp (i k x(t))},
$$

so that the average is taken over all the trajectories. Compare this formula with (8). We are led to conjecture that

$$
S\left(x, t ; V_{0}\right)=2 \pi P\left(V_{0}\right) \overline{\exp (i k x(t))},
$$

where the average, as always, is taken over the trajectories with the same $V_{0}$. Indeed, not only ( 8 ) is automatically satisfied, but also this form of $S$ reflects the fact that the particle propagates with a given energy.

With this definition of $S$ and $G$ it is easy to check that the formulae (10)-(13) are indeed true and precisely defined. Indeed, their applicability was at small $k$ where

$$
\overline{\exp (i k x(t))} \approx 1-\frac{k^{2}}{4} \overline{x^{2}(t)} \approx \exp \left(-\frac{k^{2}}{4} \overline{x^{2}(t)}\right) .
$$

From this point on, (10)-(13) can be carried on without any further obstacles. The only difference between classical and quantum case will be the value of $\nu$, which in the classical case is equal to $4 / 3$.

It is interesting to note that at large $k$ the functions $S$ and $G$ will be very different in quantum and classical case. In the quantum case, $S$ is a function of the ratio $q^{2} / \omega$. In the classical case this question was investigated in [9]. There it was shown that $S(x, t ; 0)$, computed at zero energy, was a function of $q^{\frac{7}{4}} / \omega$, with $7 / 4$ being the fractal dimension of the infinite percolating cluster. We refer the reader to [9] for further information on this subject. In the meanwhile, the form of $G$ or even $S$ at generic values of energy in the classical case remains unknown.

Finally, we would like to comment on the interesting feature of the numerical data of Fig. $⿴$. The diffusion constant, when read off this data (given in units where the bonds of the triangular lattice of Fig. 3 have unit length), is equal to

$$
D=1.008 \pm 0.014
$$

This number is remarkably close to 1

Assuming that $D=1$ is indeed an exact relation, we can demonstrate that the critical conductivity is equal to

$$
\sigma_{x x}=\frac{\sqrt{3}}{4} \frac{e^{2}}{h},
$$

an exact result recently derived in 12 by a very different approach. To do that, we need to use the relationship between the diffusion constant and the critical conductivity proposed by F. Evers and W. Brenig in [13].

According to the Einstein's relation, the conductivity is given by

$$
\sigma_{x x}=e^{2} \rho \frac{D}{4},
$$

where $e$ is the electron charge and $\rho$ is the density of states per unit area per unit energy at the transition. The problem is to find $\rho$ in the lattice model introduced here. 
We can use a standard quasiclassical argument to find the density of states for a particle moving in a magnetic field and a potential $V(x)$. The total number of states whose energy is below $E$ is given, in the quasiclassical limit, by the area of the region where $V(x)<E$, divided by $2 \pi l^{2}$,

$$
A(E)=\frac{1}{2 \pi l^{2}} \int d^{2} x \theta(E-V(x)) .
$$

The density of states per unit area is

$$
\rho(E)=\frac{1}{2 \pi l^{2} A} \int d^{2} x \delta(E-V(x)),
$$

where $A$ is the total area.

Comparing $\rho(E)$ with (31) we see that $\rho(E)$ coincides with the time $T$ it takes for a particle to go over all the trajectories with energy $E$. Restoring $\hbar$ and $l$ dependence in (31) we find

$$
\rho(E)=\frac{T}{h A}
$$

Therefore, the conductivity reduces to

$$
\sigma=\frac{e^{2}}{h} \frac{D T}{4 A}
$$

It is now clear that the overall answer is independent of the units in which we measure $D$, as long as they are the same as the ones for $A$ and $T$.

In the lattice model of Fig. 3 the total time is equal to the total number of links available for a particle with zero energy, the energy of the transition. Assume that the total number of triangles is equal to $N$. The area $A$ of the lattice is equal to $N \times \frac{\sqrt{3}}{4}$, where $\frac{\sqrt{3}}{4}$ is the area of a single triangle. The total number of links is $L=\frac{3 N}{4}$. We took into account that each triangle has 2 links with a probability $3 / 4$. This is because with a probability of $1 / 4$ all three energies at the vertices of a triangle are either less or greater than 0 , in which case there are no trajectories in the triangle at all. But each link belongs to two triangles, hence the overcounting and the answer of $\frac{3 N}{4}$. Substituting this into 46 ) and taking into account that $D \approx 1$, we arrive at

$$
\sigma_{x x}=D \frac{\sqrt{3}}{4} \frac{e^{2}}{h} \approx \frac{\sqrt{3}}{4} \frac{e^{2}}{h},
$$

in very good agreement with [12].

This result is also in agreement with the one of [13] where the critical conductivity was estimated at $\sigma_{x x} \approx 0.45 \frac{e^{2}}{h}$.

Notice that the conductance of a finite sample would not be Ohmic since the particle does not undergo a simple random walk, as reflected in a complicated structure of $G$ or $S$ at finite momenta $k$. Only for very thin samples, when it takes little time for a particle to reach one end of a sample from another, can the Ohm's law be applicable, in agreement with 12 .

\section{CONCLUSIONS}

To summarize, we showed that in the classical limit the density of a particle undergoing the quantum Hall transition moves along the percolation hull trajectories. At small times this particle exhibits normal diffusion, while at large times it localizes with the localization length exponent given by the percolation $\nu=\frac{4}{3}$. Our paper sets a new framework in which the classical percolation to quantum Hall effect renormalization group flow can be studied. Finally, we confirm numerically with a high degree of accuracy the result $\sigma_{x x}=\sqrt{3} / 4$ for the critical conductivity of a classical quantum Hall transition.

\section{ACKNOWLEDGMENTS}

The authors are grateful to S. Boldyrev for many useful discussions and comments, to F. Evers who painstakingly explained his method [13] of relating the lattice diffusion constant to conductivity to us, to J. Cardy for interesting comments and to to E. H. Rezayi for making his computer facilities available to us. 
This work was supported by the NSF grant PHY 94-07194

[1] J. Sinova, V. Meden, S Girvin, Phys. Rev. B62, 2008 (2000)

[2] J. T. Chalker, Solid State Phys. 21 (1988) L119; J. T. Chalker, G. J Daniell. Phys. Rev. Lett. 61, 593 (1988)

[3] C. Itzykson, J.-M. Drouffe, Statistical Field Theory, chapter 10

[4] S.M. Girvin and T. Jach, Phys. Rev. B29, 5617 (1984); S.M. Girvin, A.H. MacDonald, and P. M. Platzman, Phys. Rev. B33, 2481 (1986)

[5] M. Isichenko, Rev. Mod. Phys. 64, 961 (1992)

[6] S. Trugman, Phys. Rev. B27 7539 (1983)

[7] M. den Nijs, J. Phys. A 12, 1857 (1979); Phys. Rev. B 27, 1674 (1983)

[8] J. T. Chalker, P. D. Coddington, J. Phys. C 21, 2665 (1988)

[9] F. Evers, Phys. Rev. E55 (3), 2321 (1997)

[10] H. Saleur and B. Duplantier, Phys. Rev. Lett. 58, 2352 (1987)

[11] S. Boldyrev, V. Gurarie, cond-mat/0009203

[12] J. Cardy, Phys. Rev. Lett. 84, 3507 (2000)

[13] F. Evers, W. Brenig, Z. Phys. B94, 155 (1994) 